\title{
A compression line for soils with evolving particle and pore size distributions due to particle crushing
}

\author{
A. R. RUSSELL*
}

\begin{abstract}
In this study it is supposed that energy is dissipated in two ways when particle crushing occurs. The first is the release of strain energy stored in the crushed particle and is analogous to the creation of surface. The second is due to the load redistribution and change of stored strain energy of the surrounding soil. Terms for these two mechanisms are included in a Cam-clay type energy equation. By defining particle and pore size distributions during the crushing process using fractals, and by equating particle and pore surface areas, a closed-form expression is obtained for a limiting compression line in the double logarithmic voids ratio-stress plane. The limiting compression line and the evolving particle size distribution are matched well by the theory for two silica sands loaded in oedometric compression to high stresses. Evidently, energy dissipation due to load redistribution is significantly larger than energy dissipation due to the creation of surface.
\end{abstract}

KEYWORDS: compressibility; fractals; particle crushing

ICE Publishing: all rights reserved

\section{INTRODUCTION}

Many expressions have been proposed for compression lines for granular soils exhibiting particle crushing. Most are phenomenological in origin and rely on empirical fitting parameters. However, a few theoretical studies have derived closed-form expressions for compression lines. McDowell et al. (1996) and McDowell \& Bolton (1998) used a fractal description for the particle size distribution and sizedependent particle strength to define a linear compression line in the $e-\ln \sigma$ plane, where $e$ represents the voids ratio and $\sigma$ the applied stress. No reference was made to how pore sizes evolve; rather, volumetric deformation was indirectly included through an assumed energy balance. McDowell (2005) later proposed that $e$ is proportional to the volume of the smallest particles and suggested the compression line may be more appropriately defined in the $\ln e-\ln \sigma$ plane. Definition in this plane seems sensible as a straight line may continue indefinitely with increasing $\sigma$ and reducing $e$ while maintaining a positive value.

In this study, a new approach is presented to derive a theoretical expression for a limiting compression line. For the first time, fractal characteristics of the pore space are considered along with those of the particles. A more complete energy balance equation is also used, which accounts for energy dissipation through the creation of surface as well as load redistribution in the surrounding soil. The derivation is made possible by assuming many material parameters relevant to compression behaviour are constant (e.g. surface energy, fractal dimensions and shape factors for particles and pores) and is reasonable once a significant amount of crushing has occurred.

Manuscript received 17 December 2010; first decision 11 January 2011; accepted 19 January 2011.

Published online 8 February 2011.

* Centre for Infrastructure Engineering and Safety, School of Civil and Environmental Engineering, The University of New South Wales, Sydney, Australia

\section{ENERGY CONSIDERATIONS}

When a particle in a granular soil is crushed, the energy that is dissipated may be divided into two parts (Nguyen \& Einav, 2009). The first is due to release of strain energy stored in the particle and is analogous to energy associated with the creation of surface area of the fragments, denoted $\Delta \Phi_{\text {surface }}$. The second is due to load redistribution (and associated displacement) of the surrounding soil. This energy dissipation mechanism, denoted $\Delta \Phi_{\text {redist }}$, is rarely considered, although Nguyen \& Einav (2009) showed it to be significant.

Assuming that only plastic work input is dissipated, a simple link between plastic work input per unit volume and the main dissipation processes is given by

$$
\sigma_{i j} \delta \varepsilon_{i j}^{\mathrm{p}}=\Delta \Phi_{\text {friction }}+\Delta \Phi_{\text {surface }}+\Delta \Phi_{\text {redist }}
$$

This can be written in a form that is an extension of the Cam-clay equation

$$
p \delta \varepsilon_{p}^{\mathrm{p}}+q \delta \varepsilon_{q}^{\mathrm{p}}=M p \delta \varepsilon_{q}^{\mathrm{p}}+\frac{\Gamma \delta S_{\mathrm{s}}}{V_{\mathrm{s}}(1+e)}(1+R)
$$

where $\Delta \Phi_{\text {friction }}=M p \delta \varepsilon_{q}^{\mathrm{p}}, \Delta \Phi_{\text {surface }}=\Gamma \delta S_{\mathrm{s}} / V_{\mathrm{s}}(1+e)$ and $R=\Delta \Phi_{\text {redist }} / \Delta \Phi_{\text {surface. }}$. The mean stress $p$ and deviatoric stress $q$ cause plastic increments of volumetric strain $\varepsilon_{p}^{\mathrm{p}}$ and deviatoric strain $\varepsilon_{q}^{\mathrm{p}} . \Delta \Phi_{\text {friction }}$ represents dissipation through friction (primarily due to sliding between particles other than those that crush) and $M$ is a function of the internal frictional strength of the soil. $\Delta \Phi_{\text {surface }}+\Delta \Phi_{\text {redist }}$ accounts for dissipation due to particle crushing. $\Delta S_{\mathrm{s}}$ is the increase in surface area of particles within a soil having volume of solids $V_{\mathrm{s}}$ and $\Gamma$ represents the surface energy (with units of $\mathrm{N} / \mathrm{m}) . \Gamma(1+R)$ is an assumed material constant for a given load path and boundary condition (Nguyen \& Einav, 2009) as discussed later. An additional frictional dissipation belonging to newly formed fragments of a crushed particle may also exist, even for isotropic loading. Rather than using a separate dissipation term for this, it is assumed to be contained in $\Delta \Phi_{\text {surface }}$. When $R=0, \Delta \Phi_{\text {redist }}=0$ and the work equation reduces to that given by McDowell \& Bolton (1998). 
For oedometric compression, and supposing elastic strains are very small compared with plastic strains so can be neglected for simplicity (elastic compression of the solid particles and its influence on $e$ is not considered), equation (2) becomes

$$
\sigma_{\mathrm{v}} \delta \varepsilon_{p}=\frac{2}{9} M\left(1+2 K_{0}\right) \sigma_{\mathrm{v}} \delta \varepsilon_{p}+\frac{\Gamma \delta S_{\mathrm{s}}}{V_{\mathrm{s}}(1+e)}(1+R)
$$

where $\sigma_{\mathrm{v}}$ is the vertical stress and $K_{0}$ is the ratio between horizontal and vertical stresses.

\section{FRACTAL DEFINITIONS}

Fractal properties of a granular material undergoing particle crushing may now be combined with equation (3) to derive a compression line linking $e$ to $\sigma_{\mathrm{v}}$. It is supposed that the number of pores being of any size $L$ larger than $d_{\mathrm{p}}$ and the number of particles being of any size $L$ larger than $d_{\mathrm{s}}$ obey

$$
N_{\mathrm{p}}\left(L>d_{\mathrm{p}}\right) \propto d_{\mathrm{p}}^{-D_{\mathrm{p}}} \text { and } N_{\mathrm{s}}\left(L>d_{\mathrm{s}}\right) \propto d_{\mathrm{s}}^{-D_{\mathrm{s}}}
$$

where $D_{\mathrm{p}}$ and $D_{\mathrm{s}}$ are the fractal dimensions characterising the distributions of pore and particle sizes, respectively. The combined surface areas of pores $S_{\mathrm{p}}$ and particles $S_{\mathrm{s}}$ larger than size $d_{\mathrm{p}}$ and $d_{\mathrm{s}}$, respectively, then obey

$$
S_{\mathrm{p}}\left(L>d_{\mathrm{p}}\right) \propto d_{\mathrm{p}}^{2-D_{\mathrm{p}}} \text { and } S_{\mathrm{s}}\left(L>d_{\mathrm{s}}\right) \propto d_{\mathrm{s}}^{2-D_{\mathrm{s}}}
$$

The total surface areas of pores and particles obey:

$$
S_{\mathrm{pT}} \propto d_{\mathrm{p} \min }^{2-D_{\mathrm{p}}} \text { and } S_{\mathrm{sT}} \propto d_{\mathrm{s} \min }^{2-D_{\mathrm{s}}}
$$

where subscript min indicates minimum size and $\mathrm{T}$ indicates total. Russell (2010) assumed the total particle and pore surface areas are equal (supposing the combined contact area of touching particles is very small compared with the total surface area) to derive

$$
\left(\frac{d_{\mathrm{p} \min }}{d_{\mathrm{p} \max }}\right)^{2-D_{\mathrm{p}}}=\left(\frac{d_{\mathrm{s} \min }}{d_{\mathrm{s} \max }}\right)^{2-D_{\mathrm{s}}}
$$

where subscript max indicates maximum size. Equation (7) implies that a geometrical relationship exists between cumulative surface areas of pores and particles that scales proportionally to their size.

The volumes of pores $V_{\mathrm{p}}$ and particles $V_{\mathrm{s}}$ larger than $d_{\mathrm{p}}$ and $d_{\mathrm{s}}$, respectively, obey

$$
V_{\mathrm{p}}\left(L>d_{\mathrm{p}}\right) \propto d_{\mathrm{p}}^{3-D_{\mathrm{p}}} \text { and } V_{\mathrm{s}}\left(L>d_{\mathrm{s}}\right) \propto d_{\mathrm{s}}^{3-D_{\mathrm{s}}}
$$

For pores, this can be written more completely as

$$
V_{\mathrm{p}}\left(L>d_{\mathrm{p}}\right)=V_{\mathrm{pT}}\left(\frac{d_{\mathrm{p} \max }^{3-D_{\mathrm{p}}}-d_{\mathrm{p}}^{3-D_{\mathrm{p}}}}{d_{\mathrm{p} \max }^{3-D_{\mathrm{p}}}-d_{\mathrm{p} \min }^{3-D_{\mathrm{p}}}}\right)
$$

Furthermore, the particle size distribution curve, which expresses the percentage by mass of particles of size $L$ smaller than $d_{\mathrm{s}}$ as a function of $d_{\mathrm{s}}$, becomes

$$
\% M_{\mathrm{s}}\left(L<d_{\mathrm{s}}\right)=100\left(\frac{d_{\mathrm{s}}^{3-D_{\mathrm{s}}}-d_{\mathrm{s} \min }^{3-D_{\mathrm{s}}}}{d_{\mathrm{s} \max }^{3-D_{\mathrm{s}}}-d_{\mathrm{s} \min }^{3-D_{\mathrm{s}}}}\right)
$$

The total surface areas of particles or pores per total volumes of particles or pores are then given by

$$
\begin{aligned}
& \frac{S_{\mathrm{sT}}}{V_{\mathrm{sT}}}=\frac{3-D_{\mathrm{s}}}{2-D_{\mathrm{s}}} \frac{\beta_{\mathrm{sS}}}{\beta_{\mathrm{sV}}}\left(\frac{d_{\mathrm{s} \max }^{2-D_{\mathrm{s}}}-d_{\mathrm{s} \min }^{2-D_{\mathrm{s}}}}{d_{\mathrm{s} \max }^{3-D_{\mathrm{s}}}-d_{\mathrm{s} \min }^{3-D_{\mathrm{s}}}}\right) \\
& \frac{S_{\mathrm{pT}}}{V_{\mathrm{pT}}}=\frac{3-D_{\mathrm{p}}}{2-D_{\mathrm{p}}} \frac{\beta_{\mathrm{pS}}}{\beta_{\mathrm{pV}}}\left(\frac{d_{\mathrm{p} \max }^{2-D_{\mathrm{p}}}-d_{\mathrm{p} \min }^{2-D_{\mathrm{p}}}}{d_{\mathrm{p} \max }^{3-D_{\mathrm{p}}}-d_{\mathrm{p} \min }^{3-D_{\mathrm{p}}}}\right)
\end{aligned}
$$

in which $\beta_{\mathrm{sS}}$ and $\beta_{\mathrm{pS}}$ are surface area shape factors for particles or pores and $\beta_{\mathrm{sv}}$ and $\beta_{\mathrm{pV}}$ are volumetric shape factors for particles or pores. Harr (1977) reported that the ratio $\beta_{\mathrm{sS}} / \beta_{\mathrm{sV}}$ lies between 14 and 18 for particles belonging to real granular soils. $\beta_{\mathrm{sS}} / \beta_{\mathrm{sV}}$ and $\beta_{\mathrm{pS}} / \beta_{\mathrm{pV}}$ are assumed constant.

Modelling particle and pore size distributions using fractals provides a good fit to experimental data (e.g. McDowell et al., 1996; Coop et al., 2004; Einav, 2007a, 2007b; Yu et al. 2009; Russell, 2010). For particle size distributions, however, a constant $D_{\mathrm{s}}$ is reasonable only if a significant amount of particle crushing has occurred. Using equation (10) with a constant $D_{\mathrm{s}}$ to define the particle size distribution for a soil before loading (uncrushed) and its evolution to when a significant amount of crushing has occurred may not be possible. A more elaborate model that can handle this may introduce into the particle size distribution a state variable in addition to $d_{\mathrm{s} \min }$, as in Einav (2007a, 2007b). The applicability of the constant $D_{\text {s }}$ assumption, and what proves to be a significant amount of crushing, is demonstrated later. For most soils formed by fragmentation or crushing processes, $D_{\mathrm{s}}$ takes on a value between $2 \cdot 2$ and $2 \cdot 8$ (e.g. Perfect, 1997; Coop et al., 2004).

\section{DERIVATION OF A COMPRESSION LINE}

The following derivations apply to when $D_{\mathrm{s}}$ and $D_{\mathrm{p}}$ may be taken as constants (within the range 2-3) once a significant amount of crushing has occurred. It is assumed that, as soil particles crush, the maximum particle size $\left(d_{\mathrm{s} \max }\right)$ remains unaltered as the minimum particle size $\left(d_{\mathrm{s} \text { min }}\right)$ becomes smaller; this is supported by experimental data (e.g. Lade et al., 1996; Nakata et al., 2001; Luzzani \& Coop, 2002). The first part of equation (11) can then be written in rate form

$$
\frac{\delta S_{\mathrm{sT}}}{V_{\mathrm{sT}}}=\frac{\mathrm{d}\left(S_{\mathrm{sT}} / V_{\mathrm{sT}}\right)}{\mathrm{d} d_{\mathrm{s} \min }} \delta d_{\mathrm{s} \min }
$$

Recalling that the surface areas of pores and particles are assumed equal, $e$ may be written as

$$
e=\frac{V_{\mathrm{pT}}}{V_{\mathrm{sT}}}=\frac{S_{\mathrm{sT}} / V_{\mathrm{sT}}}{S_{\mathrm{pT}} / V_{\mathrm{pT}}}
$$

This will be a function of variables $d_{\mathrm{p} \text { max }}, d_{\mathrm{p} \text { min }}$ and $d_{\mathrm{s} \min } \cdot d_{\mathrm{p} \min }$ can be removed using equation (7). $d_{\mathrm{p} \max }$ can be removed using the finding of Dodds \& Weitz (2002) (further verified by Delaney et al. (2008)) that it is proportional to the total number of particles raised to the power $-\left(3-D_{\mathrm{s}}\right)$ and therefore, from equation (4), must be proportional to $d_{\mathrm{smin}}$ raised to the power $\left(3-D_{\mathrm{s}}\right) D_{\mathrm{s}}$. This can be expressed mathematically as

$$
d_{\mathrm{p} \max }=C_{1} d_{\mathrm{s} \max }\left(\frac{d_{\mathrm{s} \min }}{d_{\mathrm{s} \max }}\right)^{\left(3-D_{\mathrm{s}}\right) D_{\mathrm{s}}}
$$

in which $C_{1}$ is a dimensionless material constant. $e$ then becomes a function of $d_{\mathrm{s} \text { min }}$ only, and the rate form is

$$
\delta e=\left(\frac{\mathrm{d}\left[\left(S_{\mathrm{sT}} / V_{\mathrm{sT}}\right) /\left(S_{\mathrm{pT}} / V_{\mathrm{pT}}\right)\right]}{\mathrm{d} d_{\mathrm{s} \min }}\right) \delta d_{\mathrm{s} \min }
$$



that

Using the volumetric strain definition it can be shown

$$
\begin{aligned}
& \delta \varepsilon_{\mathrm{p}}=-\frac{\delta e}{1+e}=-\frac{1}{1+e} \\
& \times\left(\frac{\mathrm{d}\left[\left(S_{\mathrm{sT}} / V_{\mathrm{sT}}\right) /\left(S_{\mathrm{pT}} / V_{\mathrm{pT}}\right)\right]}{\mathrm{d} d_{\mathrm{s} \min }}\right) \delta d_{\mathrm{s} \min }
\end{aligned}
$$

An expression for $\sigma_{\mathrm{v}}$ then becomes

$$
\begin{aligned}
& \sigma_{\mathrm{v}}=-\left(\frac{9 \Gamma(1+R)}{9-2 M\left(1+2 K_{0}\right)}\right) \frac{\delta S_{\mathrm{sT}}}{V_{\mathrm{sT}}} \frac{1}{\delta e}= \\
& -\left(\frac{9 \Gamma(1+R)}{9-2 M\left(1+2 K_{0}\right)}\right)\left(\frac{\mathrm{d}\left(S_{\mathrm{sT}} / V_{\mathrm{sT}}\right)}{\mathrm{d} d_{\mathrm{s} \min }}\right) \\
& \times\left(\frac{\mathrm{d} d_{\mathrm{s} \min }}{\mathrm{d}\left[\left(S_{\mathrm{sT}} / V_{\mathrm{sT}}\right) /\left(S_{\mathrm{pT}} / V_{\mathrm{pT}}\right)\right]}\right)
\end{aligned}
$$

which is also a function of $d_{\mathrm{s} \min }$, so has the rate form:

$$
\delta \sigma_{\mathrm{v}}=\frac{\mathrm{d} \sigma_{\mathrm{v}}}{\mathrm{d} d_{\mathrm{s} \min }} \delta d_{\mathrm{s} \min }
$$

The compressive response is then defined by

$$
\delta e=\left(\frac{\mathrm{d}\left[\left(S_{\mathrm{sT}} / V_{\mathrm{sT}}\right) /\left(S_{\mathrm{pT}} / V_{\mathrm{pT}}\right)\right]}{\mathrm{d} d_{\mathrm{s} \min }}\right)\left(\frac{\mathrm{d} d_{\mathrm{s} \min }}{\mathrm{d} \sigma_{\mathrm{v}}}\right) \delta \sigma_{\mathrm{v}}
$$

It so happens that when $d_{\mathrm{p} \max } \gg d_{\mathrm{p} \text { min }}$ and $d_{\mathrm{s} \max } \gg d_{\mathrm{s} \text { min }}$ (which is the case once a significant amount of particle crushing has occurred), the compressive response approaches, as an asymptote, a straight line in the $\ln e-\ln \sigma_{\mathrm{v}}$ plane. This asymptote, referred to as a limiting compression line, is defined in non-dimensional form as

$$
\ln e=\ln N-\lambda \ln \frac{\sigma_{\mathrm{v}}}{\Gamma / d_{\mathrm{s} \max }}
$$

where $\lambda$ and $N$ are dimensionless material constants. $\Gamma$ and $d_{\text {smax }}$ are also material constants during the crushing process. After mathematical manipulations, the complete expressions for $\lambda$ and $N$ are

$$
\lambda=-\frac{\left(3-D_{\mathrm{s}}\right) D_{\mathrm{s}}}{2-4 D_{\mathrm{s}}+D_{\mathrm{s}}^{2}}
$$

and

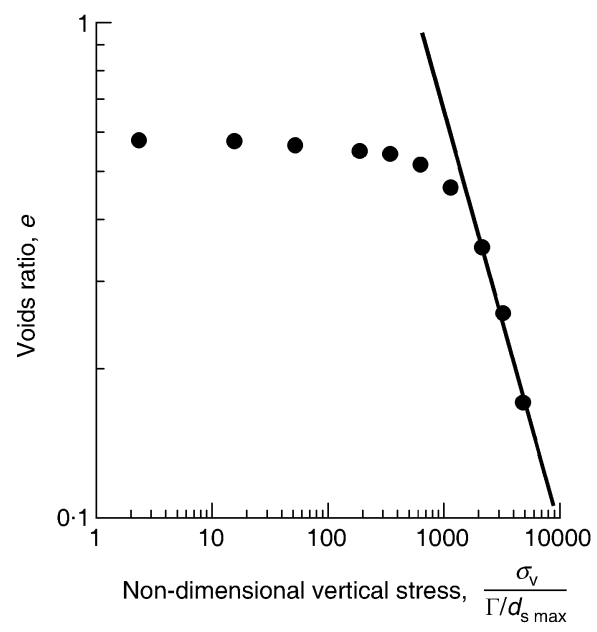

(a)

$$
\begin{aligned}
& N=\left[C_{1}\left(\frac{\left(-3+D_{\mathrm{s}}\right)\left(-2+D_{\mathrm{p}}\right)}{\left(-3+D_{\mathrm{p}}\right)\left(-2+D_{\mathrm{s}}\right)}\right) \frac{\beta_{\mathrm{pV}}}{\beta_{\mathrm{pS}}}\right]^{1-\lambda} \\
& \times\left[\frac{\beta_{\mathrm{sS}}}{\beta_{\mathrm{sV}}}\left(\frac{1}{D_{\mathrm{s}}} \frac{9(1+R)}{9-2 M\left(1+2 K_{0}\right)}\right)^{\lambda}\right]
\end{aligned}
$$

Significant simplifications would appear in the definition for $N$ when $\lambda=1$, and this would be the case when $D_{\mathrm{s}}=2 . N$ would then depend on only $\beta_{\mathrm{sS}} / \beta_{\mathrm{sV}}, R, M$ and $K_{0}$, which would be more or less constant for all granular soil types in oedometric compression and independent of pore properties.

It is noted that $\Gamma$ and $R$ may actually depend on load path and stress state as well as particle size and particle contact geometry, although there have been very few studies in this area. One example (Nguyen \& Einav, 2009) used a 1D model of linear springs and breakable connectors to motivate an $R$ value depending on the average number of particles along a single force chain. The length of the chain is dependent on stress and particle size. In any case, it is worth attempting to validate eqs (20), (21) and (22) for constant $\Gamma$ and $R$ and oedometric compression.

\section{VALIDATION}

Oedometric compression data and evolving particle size distributions for two sands comprising different sizes of silica sand particles were considered (Nakata et al., 2001). Before crushing, sand 1 contained particle sizes that ranged between 0.25 and $2 \mathrm{~mm}$; sand 2 particles were in the range $1 \cdot 4-1.7 \mathrm{~mm}$. Fitting only compression data is unsatisfactory as there would be no check on the evolution of $d_{\mathrm{s} \min }$. Ideally, the evolving pore size distributions would also feature in the validations, but data with which to do this are not available.

The compression data and theoretical limiting compression lines are presented in the $\ln e-\ln \left[\sigma_{\mathrm{v}} /\left(\Gamma / d_{\mathrm{s} \max }\right)\right]$ plane in Figs 1(a) and 2(a). Measured particle size distribution curves for the later stages of loading along with those predicted by equation (10) are shown in Figs 1(b) and 2(b).

Table 1 summarises the material parameters used. The same values of $D_{\mathrm{s}}, D_{\mathrm{p}}, \beta_{\mathrm{sS}} / \beta_{\mathrm{sV}}, \beta_{\mathrm{pS}} / \beta_{\mathrm{pV}}, M$ and $K_{0}$ were assumed to apply to both sands. These were selected based on typical values, except for $D_{\text {s }}$ which was found by fitting equation (10) to the particle size distribution curves and equation (21) to the asymptotic slopes of the compression

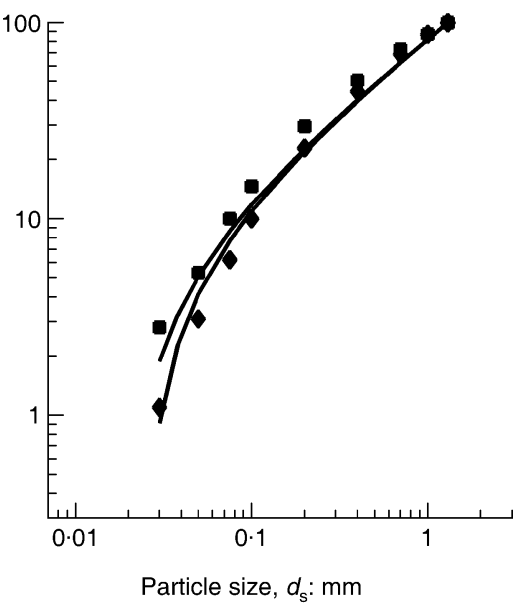

(b)

Fig. 1. Sand 1: (a) oedometric compression data; (b) particle size distribution. The symbols represent the experimentally measured data and the curves represent theoretical simulations. In (b), the diamond and square symbols indicate loading to $\sigma_{\mathrm{v}}=62$ and $92 \mathrm{MPa}$, respectively 


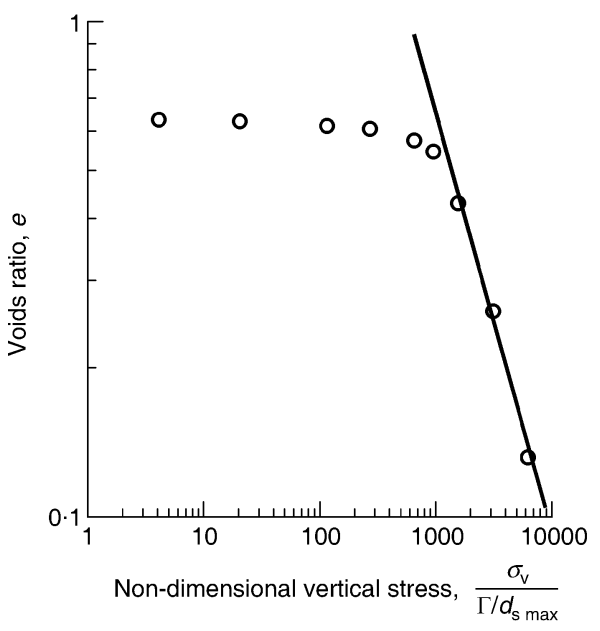

(a)

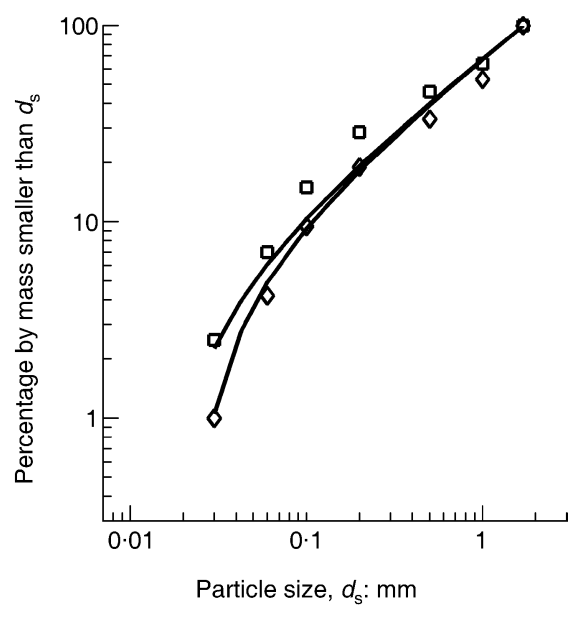

(b)

Fig. 2. Sand 2: (a) oedometric compression data; (b) particle size distribution. The symbols represent the experimentally measured data and the continuous lines represent theoretical simulations. In (b), the diamond and square symbols indicate loading to $\sigma_{\mathrm{v}}=46$ and $92 \mathrm{MPa}$, respectively

lines. The different initial particle size distributions for the two sands meant that different values of $d_{\text {smax }}$ were used. A typical value of $\Gamma=25 \mathrm{~N} / \mathrm{m}$ was assumed to apply to both sands. Finally, parameters $R$ and $C_{1}$ were found through their influence on $N$ and by fitting equation (20) to the asymptotic compression lines. It was necessary to adopt different $R$ and $C_{1}$ values for each sand to ensure a close match between theoretical and experimental $\sigma_{\mathrm{v}}$ and $e$ values. It was also necessary to select appropriate values of $d_{\text {smin }}$ that provide agreement between theory and experiment. The adopted values and the corresponding values of $\sigma_{\mathrm{v}}$ and $e$, both experimental and theoretical, are summarised in Table 2.

As $\lambda$ was close to unity, $C_{1}, D_{\mathrm{p}}$ and $\beta_{\mathrm{pS}} / \beta_{\mathrm{pV}}$ have only a minor influence on $N ; \beta_{\mathrm{sS}} / \beta_{\mathrm{sV}}, R, M$ and $K_{0}$ have a major influence. Of these parameters, $R$ cannot be measured directly in independent experiments. However, this study indicates that for oedometric compression, typical values of $\Gamma(1+R)$ are around $400 \mathrm{~N} / \mathrm{m}$, and that energy dissipation due to load redistribution has a much greater influence than that due to the creation of surface.

The $N$ values turned out to be almost identical for the two sands considered, as the differences in $C_{1}$ were offset by the differences in $R$. A unique limiting compression line would then emerge if $C_{1}$ and $R$ were included in the nondimensionalising stress term along with $\Gamma$ and $d_{\text {smax }}$. Consideration of a wider range of experimental data is necessary to conclude whether this is coincidental or an inherent feature of compression behaviour.

Table 1. Material properties

\begin{tabular}{l|c|c}
\hline & Sand 1 & Sand 2 \\
\hline$D_{\mathrm{s}}$ & $2 \cdot 3$ & $2 \cdot 3$ \\
$D_{\mathrm{p}}$ & $2 \cdot 5$ & $2 \cdot 5$ \\
$\beta_{\mathrm{sS}} / \beta_{\mathrm{sV}}$ & 16 & 16 \\
$\beta_{\mathrm{pS}} / \beta_{\mathrm{pV}}$ & 16 & 16 \\
$d_{\mathrm{smax}}: \mathrm{m}$ & $1 \cdot 3 \times 10^{-3}$ & $1 \cdot 7 \times 10^{-3}$ \\
$M$ & $1 \cdot 2$ & $1 \cdot 2$ \\
$K_{0}$ & $0 \cdot 5$ & $0 \cdot 5$ \\
$\Gamma: \mathrm{N} / \mathrm{m}$ & 25 & 25 \\
$R$ & $15 \cdot 4$ & $13 \cdot 4$ \\
$C_{1}$ & 62 & 110 \\
$\lambda$ (from equation 21) & $0 \cdot 84$ & $0 \cdot 84$ \\
$N$ (from equation 22) & 225 & 221 \\
\hline
\end{tabular}

A good match between theoretical and experimental particle size distribution curves was only possible for data points having $e$ values less than $0 \cdot 26$; this implies that $e=0 \cdot 26$ represents an (approximate) limit below which the amount of crushing becomes significant enough for the fractal dimensions and other parameters to be held constant. The experimentally observed soil response approached a state of limiting compression for $e<0 \cdot 26$. Based on the data considered here, attempting to detect a limiting compression line for $e>0 \cdot 26$, as was done by Pestana \& Whittle (1995) and McDowell (2005), may lead to smaller (and non-unique) values of $\lambda$.

\section{CONCLUSION}

Separate energy dissipation mechanisms for the creation of surface and for load redistribution have been included in a Cam-clay type energy equation. Combining this equation with fractal definitions for evolving particle and pore size distributions led to a closed-form expression for a linear limiting compression line in the $\ln e-\ln \left[\sigma_{\mathrm{v}} /\left(\Gamma / d_{\mathrm{s} \max }\right)\right]$ plane. A point on the line corresponds to a characteristic smallest particle size, thus providing a direct link to the particle size distribution.

The slope of the compression line is a function of the fractal dimension of the particle size distribution. The position of the line is a function of particle and pore fractal dimensions, along with the shapes of particles and pores, maximum particle size, frictional strength, a surface energy constant, a dimensionless constant linking maximum pore

Table 2. Experimental and theoretical parameters relevant to particle size distributions

\begin{tabular}{l|c|c|c|c}
\hline \multirow{2}{*}{$d_{\mathrm{s} \text { min }}: \mathrm{mm}$} & \multicolumn{2}{|c|}{ Experimental } & \multicolumn{2}{c}{ Theoretical } \\
\cline { 2 - 5 } & $\begin{array}{c}\sigma_{\mathrm{v}}: \\
\mathrm{MPa}\end{array}$ & $e$ & $\begin{array}{c}\sigma_{\mathrm{v}}: \mathrm{MPa} \\
\text { (equation 17) }\end{array}$ & $e$ (equation 20) \\
\hline Sand 1 & 62 & $0 \cdot 259$ & $61 \cdot 6$ & $0 \cdot 250$ \\
$0 \cdot 025$ & 92 & $0 \cdot 171$ & $94 \cdot 3$ & $0 \cdot 174$ \\
$0 \cdot 020$ & & & & \\
Sand 2 & 46 & $0 \cdot 260$ & $45 \cdot 6$ & $0 \cdot 252$ \\
$0 \cdot 023$ & 92 & $0 \cdot 132$ & $96 \cdot 9$ & $0 \cdot 133$ \\
$0 \cdot 0155$ & &
\end{tabular}


size to particle size and the ratio of load redistribution energy to surface energy dissipation.

The compression line was matched well by two silica sands loaded in oedometric compression. The data considered suggest that energy dissipation due to load redistribution is significantly greater than that due to the creation of surface.

\section{REFERENCES}

Coop, M. R., Sorensen, K. K., Bodas Freitas, K. K. \& Georgoutsos, G. (2004). Particle breakage during shearing of a carbonate sand. Géotechnique 54, No. 3, 157-163.

Delaney, G. W., Hutzler, S. \& Aste, T. (2008). Relation between grain shape and fractal properties in random Apollonian packing with grain rotation. Phys. Rev. Lett. 101, 120602.

Dodds, P. S. \& Weitz, J. S. (2002). Packing-limited growth. Phys. Rev. E 65, 056108.

Einav, I. (2007a). Breakage mechanics - part I: Theory. J. Mech. Phys. Solids 55, No. 6, 1274-1297.

Einav, I. (2007b). Breakage mechanics - part II: Modelling of granular materials. J. Mech. Phys. Solids 55, No. 6, 1298-1320.

Harr, M. E. (1977). Mechanics of Particulate Media. New York: McGraw-Hill.

Lade, P. V., Yamamuro, J. A. \& Bopp, P. A. (1996). Significance of particle crushing in granular materials. J. Geotech. Engng ASCE 22, No. 4, 309-316.

Luzzani, L. \& Coop, M. R. (2002). On the relation between particle breakage and the critical state of sands. Soils and Found. 42, No. 2, 71-82.

McDowell, G. R. (2005). A physical justification for $\log e-\log \sigma$ based on fractal crushing and particle kinematics. Géotechnique 55, No. 9, 697-698.

McDowell, G. R. \& Bolton, M. D. (1998). On the micro mechanics of crushable aggregates. Géotechnique 48, No. 5, 667-679.

McDowell, G. R., Bolton, M. D. \& Robertson, D. (1996). The fractal crushing of granular materials. J. Mech. Phys. Solids 44, No. 12, 2079-2102.

Nakata, Y., Hyodo, M., Hyde, A. F. L., Kato, Y. \& Murata, H. (2001). Microscopic particle crushing of sand subjected to high pressure one-dimensional compression. Soils and Found. 41, No. 1, 69-82.

Nguyen, G. \& Einav, I. (2009). The energetic of cataclasis based on breakage mechanics. Pure Appl. Geophy. 166, No. 10-11, 16931724.

Perfect, E. (1997). Fractal models for the fragmentation of rocks and soils: a review. Engng Geol. 48, No. 3-4, 185-198.

Pestana, J. M. \& Whittle, A. J. (1995). Compression model for cohesionless soils. Géotechnique 45, No. 4, 611-631.

Russell, A. R. (2010). Water retention characteristics of soils with double porosity. Eur. J. Soil Sci. 61, No. 3, 412-424.

Yu, B., Cai, J. \& Zou, M. (2009). On the physical properties of apparent two-phase fractal porous media. Vadose Zone J. 8, No. 1, 177-186.

\section{WHAT DO YOU THINK?}

To discuss this paper, please email up to 500 words to the editor at journals@ice.org.uk. Your contribution will be forwarded to the author(s) for a reply and, if considered appropriate by the editorial panel, will be published as a discussion. 